\title{
THE AREA OF AN ANALYTIC SET IN COMPLEX PROJECTIVE SPACE
}

\author{
PAUL R. THIE
}

This note consists of a proof of the fact that the area of an analytic set in complex projective space is equal to the product of the degree of the set times a constant, the constant being dependent only on the dimension of the analytic set. A proof of this theorem is sketched by de Rham in [1 ]. The proof given here is quite distinct, and is a modification of Proposition 2.4 of [2].

Let $W$ be an $(n+1)$-dimensional complex vector space, $P(W)$ the associated projective space, and $A$ a $q$-dimensional analytic set in $P(W)$. Let $\omega_{q}$ be the $2 q$-dimensional volume element in $P(W)$. For a nonnegative integer $p$, let $c(p)=p ! / \pi^{p}$.

Theorem. Let $\operatorname{deg} A$ denote the degree of $A$. Then

$$
c(q) \int_{A} \omega_{q}=\operatorname{deg} A .
$$

Proof. By induction on $d=n-q$. Clearly it is sufficient to prove the theorem for $A$ irreducible. Now if $d=0, A=P(W)$ and the theorem is trivial. Assume the theorem is true for $n-q \leqq d-1$. Let $A$ be a $q$-dimensional analytic set in $P(W)$, where $W$ is an $(n+1)$-dimensional complex vector space, and where $n-q=d \geqq 1$. If $n=1, q=0$ and the result follows. Thus assume $n \geqq 2$.

Let $G$ be the Grassman manifold of $(n-q)$-dimensional planes in $P(W)$. For an arbitrary set $S$, let $\# S$ denote the number of elements of $S$, setting $\# S=0$ if $S=\varnothing$ and $\# S=\infty$ if $S$ is infinite. Then $L \in G$ and $\#\{L \cap A\}<\infty$ implies that $\#\{L \cap A\} \leqq \operatorname{deg} A$. Moreover, there exists a thin analytic set $T$ in $G$ such that $L \in G-T$ implies that $\#\{L \cap A\}$ $=\operatorname{deg} A$. Take any $L \in G-T$. Take any point $a \in L-A$. Choose an orthonormal basis of $W$ in such a way that if $W$ is identified with $C^{n+1}$ and $P(W)$ with $P\left(C^{n+1}\right)=P^{n}$, and if $\rho: C^{n+1}-\{0\} \rightarrow P^{n}$ is the residual map, then $b=(1,0, \cdots, 0) \in C^{n+1}$ is in $\rho^{-1}(a)$, and $\rho^{-1}(L)$ $=\left\{\left(z_{0}, \cdots, z_{n-q}, 0, \cdots, 0\right) \mid\left(z_{0}, \cdots, z_{n-q}\right) \neq 0\right\}$. Let $P^{n-1}=P\left(C^{n}\right)$ and $\tau: C^{n}-\{0\} \rightarrow P^{n-1}$ the residual map. Define $\pi: P^{n}-\{a\} \rightarrow P^{n-1}$ by $\pi\left(\rho\left(z_{0}, \cdots, z_{n}\right)\right)=\tau\left(z_{1}, \cdots, z_{n}\right)$. Let $\pi(A)=B$. Proposition 2.4 of [2] yields the following result: "The map $\pi \mid A: A \rightarrow B$ is proper and light, $B$ is an irreducible $q$-dimensional analytic set in $P^{n-1}$ and there exists an analytic set $S \subset B$, thin in $B$, such that $\pi \mid A-\pi^{-1}(S): A$

Received by the editors March 28, 1968. 
$-\pi^{-1}(S) \rightarrow B-S$ is a regular covering map of the complex manifold $A-\pi^{-1}(S)$ onto the connected manifold $B-S$, with covering number $\lambda$. Moreover, if $\omega_{q}^{\prime}$ is the volume element of $P^{n-1}$, then $c(q) \int_{A} \omega_{q}$ $=\lambda c(q) \int_{B} \omega_{a}^{\prime}$ holds."

Now let $\pi(L-\{a\})=L^{\prime}$. Then $L^{\prime}$ is an $(n-q-1)$-dimensional

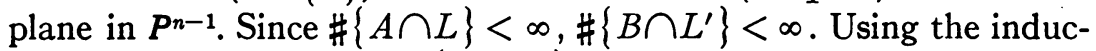
tion hypothesis, we have $\#\left\{B \cap L^{\prime}\right\} \leqq \operatorname{deg} B=c(q) \int_{B} \omega_{q}^{\prime}$. But for any $x \in B, \#\left\{\pi^{-1}(x) \cap A\right\} \leqq \lambda$. Therefore $\operatorname{deg} A=\#\{A \cap L\} \leqq \lambda\left(\#\left\{B \cap L^{\prime}\right\}\right)$ $\leqq \lambda c(q) \int_{B} \omega_{q}^{\prime}=c(q) \int_{A} \omega_{q}$.

On the other hand, let $G^{\prime}$ be the Grassman manifold of $(n-q-1)$ dimensional planes in $P^{n-1}$. Since $S$ is an analytic set in $P^{n-1}$ of dimension at most $q-1$, from Lemma A.1 of [2], there exists a thin analytic set $T^{\prime}$ in $G^{\prime}$ such that for all $M^{\prime} \in G^{\prime}-T^{\prime}$, we have $M^{\prime} \cap S=\varnothing$. Thus we may choose an $(n-q-1)$-dimensional plane $M^{\prime}$ in $P^{n-1}$ such that $M^{\prime} \cap S=\varnothing$ and $\operatorname{deg} B=\#\left\{M^{\prime} \cap B\right\}$. Let $M=\pi^{-1}\left(M^{\prime}\right) \cup\{a\}$. Then $M$ is an $(n-q)$-dimensional plane in $P^{n}$ such that $\#\{A \cap M\}<\infty$, and each point of $M^{\prime} \cap B$ has exactly $\lambda$ distinct pre-images in $M \cap A$. Thus, again using the induction assumption, we have $c(q) \int_{A} \omega_{q}=\lambda c(q) \int_{B} \omega_{q}^{\prime}$ $=\lambda\left(\#\left\{M^{\prime} \cap B\right\}\right)=\#\{M \cap A\} \leqq \operatorname{deg} A$.

Hence $c(q) \int_{A} \omega_{q}=\operatorname{deg} A$.

\section{BiBLIOGRAPHY}

1. G. de Rham, Currents in an analytic complex manifold, Seminars on Analytic Functions, Vol. 1, Inst. for Advanced Study, Princeton, N. J., 1957, pp. 54-64.

2. P. Thie, The Lelong number of a point of a complex analytic set, Math. Ann. 172 (1967), 269-312.

\section{Boston College}

